Bio - grafía. Escritos sobre la Biología y su Enseñanza. ISSN 2027-1034

Edición Extraordinaria. p.p. 957 - 965

Memorias del IX Encuentro Nacional de Experiencias en Enseñanza de la Biología y la Educación Ambiental. IV Congreso Nacional de Investigación en Enseñanza de la Biología.

\title{
ACTIVID ADES PRÁCTICAS QUE PROPICIAN EL APRENDIZAJE DEL CONCEPTO DE MICROBIOLOGÍA EN EL AULA
}

\author{
Zambrano Álvarez Daniela Paola ${ }^{1}$ \\ Álvarez Gómez Jeimy Stefany ${ }^{2}$
}

\section{RESUMEN}

El presente trabajo se desarrolló bajo el marco de la investigación cualitativa abordando como eje principal actividades prácticas como elemento para el aprendizaje del concepto de microbiología, mediante la implementación de la unidad didáctica "El mundo microscópico" en la Institución Educativa Distrital Isla del Sol, en Bogotá, con un grupo de 33 estudiantes de Quinto grado de primaria, las sesiones se desarrollaron en el enfoque de Aprendizaje Significativo. En la implementación de la unidad se desarrollaron diferentes actividades como la elaboración de esquemas, observaciones, práctica de laboratorio y explicaciones de los microorganismos. Como resultado de dichas prácticas, se encontró que los estudiantes establecen la relación entre microorganis mos y el medio donde éstos se desarrollan en relación a su contexto cotidiano.

PALABRAS CLAVE: Microorganismos, importancia y aprendizaje significativo.

\section{OBJETIVO:}

Evidenciar la funcionalidad de las actividades prácticas como una herramienta elemental para el aprendizaje del concepto de microbiología a estudiantes de quinto grado de primaria en la I.E.D. Isla del Sol.

\section{MARCO TEÓRICO}

En la enseñanza de la microbiología se presentan dificultades para explicar los diferentes fenómenos biológicos involucrados en los organismos microscópicos, puesto que trae implícito la introducción de nuevos términos para la explicación de sus temáticas. Por ello nos remitimos a la revisión de trabajos prácticos como estrategia para la enseñanza de

${ }^{1}$ dpzambranoa@correo.udistrital.edu.co. Estudiantes de Licenciatura en Biología, Universidad Distrital Francisco José de Caldas, Facultad de Ciencias y Educación.

2 isalvarezg@correo.udistrital.edu.co. Estudiantes de Licenciatura en Biología, Universidad Distrital Francisco José de Caldas, Facultad de Ciencias y Educación. 
Bio - grafía. Escritos sobre la Biología y su Enseñanza. ISSN 2027-1034

\section{Edición Extraordinaria. p.p. 957 - 965}

Memorias del IX Encuentro Nacional de Experiencias en Enseñanza de la Biología y la

Educación Ambiental. IV Congreso Nacional de Investigación en Enseñanza de la Biología.

las ciencias, lo cual, acorde con lo expuesto por Del Carmen (2000) estos trabajos pueden ser entendidos como las actividades de enseñanza de las ciencias en las que los alumnos han de utilizar determinados procedimientos que en un sentido más amplio pueden permitir el acercamiento a la resolución de problemas científicos.

En concordancia, asumimos la práctica no en función de laboratorio en sí misma sólo como los procedimientos realizados en un laboratorio, como lo expone Hodson (1994),no se trata de que los alumnos aprendan a seguir una metodología e interpretar resultados, sino que las prácticas pueden abordarse en el marco de "Cualquier método de aprendizaje que exija a los aprendices ser activos en lugar de pasivos concuerda con la idea de que los estudiantes aprenden mejor a través de la experiencia directa, por lo que podría ser descrito como «trabajo práctico»" (Hodson, 1994, p. 305).

En este sentido Rozo, (2011), plantea que el diseño de actividades prácticas deben tener una estructura, donde se debe tener en cuenta el papel que desempeña la observación, la pregunta, la teoría, los recursos y la interacción entre el maestro-estudiante para que así exista una construcción de conocimientos disciplinares, pedagógicos, epistemológicos, didácticos del contenido, del contexto y psicológicos, que permitan o generen en el alumnado la interpretación e importancia del saber científico. Asimismo se pretende promover las actividades demostrativas o prácticas, con el objetivo de que los estudiantes puedan tener un primer acercamiento al concepto de microbiología además de que puedan relacionarlo con actividades de su diario vivir, priorizando la acción del aprendizaje en la propia práctica que evidencie lo aprendido de la teoría, permitiendo al estudiante contextualizar el conocimiento teórico con la cotidianidad de su entorno.

Como fundamento de lo anteriormente expuesto, Durango (2011) plantea que para lograr que el estudiante realice un ejercicio de aprendizaje y reflexión sobre lo enseñado, es necesario la indagación sobre los conceptos previos que se relacionan con la nueva información, a manera de estructura cognitiva haciendo alusión a lo contemplado por Ausubel "El factor más importante que influye en el aprendizaje, es lo que el alumno sabe".

Sin embargo, aunque la indagación sobre las ideas previas es necesaria, ésta no contempla la totalidad de las bases para la enseñanza de las ciencias en el marco de la enseñanza científica, que siendo ésta situada en el contexto de una sociedad en la que sobra información y faltan marcos conceptuales para interpretar dicha información, tiene como base fundamental la enseñanza de datos ya que no se puede enseñar ciencias sin datos, aunque, no se debe limitar a la mera transmisión de éstos (Pozo \& Gómez, 2006). Así, al practicar la ciencia desde una postura reflexiva: el conocimiento y la habilidad que se tienen en un momento concreto determinan en el alumno la dirección de la investigación y, al mismo tiempo, el hecho de intervenir en una investigación (y lo que es decisivo, reflexionar sobre ella) hace que el conocimiento mejore y que nuestra pericia relativa al procedimiento se perfeccione (Hodson, 1994).

Además de la dificultad en la enseñanza de la microbiología las concepciones alternativas (Pozo \& Gómez, 2006), sobre las características de lo vivo a mediano plazo, pueden 
Bio - grafía. Escritos sobre la Biología y su Enseñanza. ISSN 2027-1034

\section{Edición Extraordinaria. p.p. 957 - 965}

Memorias del IX Encuentro Nacional de Experiencias en Enseñanza de la Biología y la

Educación Ambiental. IV Congreso Nacional de Investigación en Enseñanza de la Biología.

generar dificultades en aprendizaje del conocimiento científico como lo plantean Pozo \& Gómez (2006):

"El principal problema al que se enfrenta esa comprensión es la existencia en los alumnos de fuertes concepciones alternativas a los conceptos científicos que se les enseñan, que resultan muy difíciles de modificar y que en algunos casos sobreviven a largos años de instrucción científica".

\section{METODOLOGÍA}

El proyecto planteado se desarrolló desde el enfoque investigativo cualitativo. Este enfoque estudia la calidad de las actividades, relaciones, asuntos, medios, materiales o instrumentos en una determinada situación o problema (Torres, 1998), la investigación pretende hacer un análisis a un tema en particular que para el caso de este proyecto es el trabajo práctico como herramienta para el aprendizaje significativo de concepto microbiología. La investigación comprendió las siguientes fases:

a. Fase de reconocimiento y caracterización de la Institución, docente y estudiantes: Se realizó la caracterización de la I.E.D. Distrital Isla Del Sol, por medio de una descripción del PEI y de la identificación del enfoque pedagógico, posteriormente se realizó la caracterización del docente por medio de una encuesta sobre su ejercicio profesional, caracterización que se realizó también a los estudiantes mediante una encuesta de tipo socio-económico con el fin de planear las actividades en concordancia con la unidad.

b. Fase de diseño de la Unidad Didáctica: Posteriormente con los resultados obtenidos en la caracterización, se diseñó una unidad didáctica, centrada en la enseñanza del concepto de microbiología en la que se estructuraban todos los temas y contenidos que se tratarían en clase (Fonseca, 2012).

c. Fase de recolección de información: La recolección de los datos se realizó principalmente por medio de descripción de las sesiones caracterizando técnicas como la observación, talleres y práctica de laboratorio.

\section{RESULT ADOS Y ANÁLISIS}

Para la organización de los resultados se clasificaron por temáticas trabajadas en clase y niveles de caracterización según la respuesta de los estudiantes en cada sesión estructurados de la siguiente manera:

\section{TRABAJO PRÁCTICO}

Tabla No. 1: Descripción de las actividades desarrolladas en clase. 
Bio - grafía. Escritos sobre la Biología y su Enseñanza. ISSN 2027-1034

\section{Edición Extraordinaria. p.p. 957 - 965}

Memorias del IX Encuentro Nacional de Experiencias en Enseñanza de la Biología y la

Educación Ambiental. IV Congreso Nacional de Investigación en Enseñanza de la Biología.

\begin{tabular}{|c|c|c|}
\hline Temática & Categoría & Resultado y preguntas orientadoras \\
\hline$I y \mathrm{VIII}$ & Conceptual & ¿Qué son los microorganismos? \\
\hline $\begin{array}{l}\text { Microbiología } \\
\text { y } \\
\text { microorganis } \\
\text { mos. } \\
\text { Construcción } \\
\text { grupal. }\end{array}$ & $\begin{array}{l}\text { El estudiante } \\
\text { reconoce la } \\
\text { microbiología } \\
\text { como la } \\
\text { ciencia que } \\
\text { estudia los } \\
\text { microorganis } \\
\text { mos, así } \\
\text { como de su } \\
\text { morfología y } \\
\text { su } \\
\text { importancia a } \\
\text { nivel } \\
\text { biológico, } \\
\text { industrial y } \\
\text { de salud. }\end{array}$ & $\begin{array}{l}\text { El primer trabajo práctico desarrollado en clase consistió en la } \\
\text { confrontación de las ideas previas, los microorganismos, su hábitat } \\
\text { y la importancia de los mismos; con el fin de complementar se } \\
\text { realizó una breve intervención teórica, ejercicio que en sí permitió } \\
\text { al estudiante una construcción de saberes, de igual manera las } \\
\text { preguntas orientadoras y aquellas que surgieron en la clase dieron } \\
\text { paso a la contextualización de los estudiantes con la explicación del } \\
\text { tema contextualizándolo con actividades cotidianas como el manejo } \\
\text { del agua en casa, descomposición de los alimentos y } \\
\text { enfermedades como la gripa. Algunas de las concepciones } \\
\text { alternativas propuestas por los estudiantes, son la percepción } \\
\text { macroscópica de la presencia o ausencia de los microorganismos, } \\
\text { como expone Camila de } 14 \text { años "las heridas son bacterias". }\end{array}$ \\
\hline$I I, I I I$ y VI & Descriptiva & $\begin{array}{l}\text { ¿En cuál de estas imágenes podremos encontrar } \\
\text { microorganismos? }\end{array}$ \\
\hline $\begin{array}{l}\text { Tipos de } \\
\text { microorganis } \\
\text { mos. } \\
\text { Relación } \\
\text { entre } \\
\text { microorganis } \\
\text { mo y medio. }\end{array}$ & $\begin{array}{l}\text { El estudiante } \\
\text { describe los } \\
\text { tipos de } \\
\text { microorganis } \\
\text { mos y } \\
\text { característica } \\
\text { s } \\
\text { morfológicas } \\
\text { así como } \\
\text { establece la } \\
\text { relación entre } \\
\text { éste y el } \\
\text { medio donde } \\
\text { se encuentra. }\end{array}$ & $\begin{array}{l}\text { La mayoría de los estudiantes respondieron que estos organismos } \\
\text { habitan en el agua ya que reconocen los protozoos como } \\
\text { microorganismos, entendiendo la relación directamente con ciertos } \\
\text { medios y dadas condiciones características, sin embargo algunos } \\
\text { estudiantes manifestaron confusión entre los organismos como lo } \\
\text { manifiesta Brian "los protozoos son bacterias verdes". A partir de } \\
\text { estas concepciones se socializó la morfología y estructuras } \\
\text { características de cada grupo de microorganismos, permitiendo } \\
\text { una actitud participativa por parte de los estudiantes, en donde } \\
\text { relacionaron los microorganismos con su medio y con lo que ellos } \\
\text { conocen e intuyen sobre estos; muchos de ellos determinaron el } \\
\text { hábitat de los organismos por el tipo de estructuras que } \\
\text { presentaban. } \\
\text { Pregunta orientadora: ¿Qué tipos de microorganismos existen? } \\
\text { Los estudiantes relacionaron los microorganismos existentes con } \\
\text { protozoos ya que éste fue un componente que se les había } \\
\text { explicado antes de nuestra intervención. Luego de una breve } \\
\text { explicación y preguntas referentes al tema, agregaron bacterias y } \\
\text { hongos tomando como referente la descomposición de la comida, } \\
\text { como el pan. Asimismo se hizo la identificación de qué organismos } \\
\text { y qué estructuras subcelulares se pueden abarcar en el estudio de }\end{array}$ \\
\hline
\end{tabular}


Bio - grafía. Escritos sobre la Biología y su Enseñanza. ISSN 2027-1034

\section{Edición Extraordinaria. p.p. 957 - 965}

Memorias del IX Encuentro Nacional de Experiencias en Enseñanza de la Biología y la Educación Ambiental. IV Congreso Nacional de Investigación en Enseñanza de la Biología.

\begin{tabular}{|c|c|c|}
\hline & & $\begin{array}{l}\text { la microbiología como algunas algas y virus. Éstos últimos siendo } \\
\text { visibles con microscopio electrónico. } \\
\text { ¿Qué microorganismos identifica en las diferentes muestras } \\
\text { observadas en el laboratorio? } \\
\text { Los estudiantes realizaron dibujos sobre los microorganismos } \\
\text { observados en clase, en los cuales se identificaron que la mayoría } \\
\text { de los estudiantes tienen una compresión de las diferentes formas } \\
\text { que presentan los microorganismos como protozoos y hongos } \\
\text { unicelulares, formas de bacterias como bacilos y cocos "se puede } \\
\text { ver unas bolitas moradas que son los cocos que forman la bacteria, } \\
\text { es stafilococo"(respuesta de un estudiante); es importante resaltar } \\
\text { que la mayoría de los estudiantes ya conocían el uso y pero no el } \\
\text { manejo de los microscopios dificultando las práctica de laboratorio. } \\
\text { Los estudiantes describieron en sus dibujos tanto la forma como el } \\
\text { color que presentaban los microorganismos que se observaron, la } \\
\text { mayoría de ellos presentaron dificultades en las muestras por el } \\
\text { manejo del microscopio. }\end{array}$ \\
\hline $\begin{array}{l}\text { VII } \\
\text { Identificación } \\
\text { morfológica. } \\
\text { Estructuras y } \\
\text { funciones }\end{array}$ & $\begin{array}{l}\text { Funcional } \\
\text { El estudiante } \\
\text { describe y } \\
\text { reconoce la } \\
\text { funcionalidad } \\
\text { de las } \\
\text { estructuras } \\
\text { presentes en } \\
\text { los } \\
\text { microorganis } \\
\text { mos con } \\
\text { relación al } \\
\text { medio donde } \\
\text { se desarrolla. }\end{array}$ & $\begin{array}{l}\text { ¿En qué clase de organismos pueden ser huéspedes los } \\
\text { microorganismos? } \\
\text { Esta categoría se realizó con el fin de dar a conocer a los } \\
\text { estudiantes que los microorganismos se encuentran en diferentes } \\
\text { huéspedes y otros que presentan vida libre, resaltando la } \\
\text { importancia del lavado de manos e higiene del cuerpo, para } \\
\text { prevenir que ciertos microorganismos puedan causar } \\
\text { enfermedades. En esta parte a los estudiantes se les pidió escoger } \\
\text { diferentes fichas con imágenes que ellos consideran fundamentales } \\
\text { para la construcción de un esquema, el } 70 \% \text { de los estudiantes } \\
\text { relacionan los microorganismos como un agente infeccioso en } \\
\text { donde son causantes de enfermedad. }\end{array}$ \\
\hline
\end{tabular}

Las temáticas I y VIII se reconocieron como categoría conceptual ya que los estudiantes interpretaron, analizaron y reflexionaron lo comprendido acerca de los microorganismos y su ambiente a partir de la construcción de las diferentes actividades, en donde se identificó que el $90 \%$ de los estudiantes reconocen que dichos organismos se pueden encontrar en cualquier parte de la naturaleza como en los ecosistemas, en el suelo, en el aire, en algunos animales en estado de descomposición, en el agua y en los alimentos, esto concuerda con los resultados expuestos por Rozo (2011): 
Bio - grafía. Escritos sobre la Biología y su Enseñanza. ISSN 2027-1034

\title{
Edición Extraordinaria. p.p. 957 - 965
}

\author{
Memorias del IX Encuentro Nacional de Experiencias en Enseñanza de la Biología y la \\ Educación Ambiental. IV Congreso Nacional de Investigación en Enseñanza de la \\ Biología.
}

"los estudiantes afirmaron que los microorganismos también se encuentran en ecosistemas acuáticos y terrestres, dando a entender que no solo en la organización externa de los seres vivos de un ecosistema se encuentran dichos organismos sino que también a nivel interno se pueden encontrar" (p. 9).

Posterior al trabajo práctico se socializó la explicación de cada grupo a la clase en general. Al finalizar la primera sesión se dejó como complemento la elaboración de un esquema que relaciona lo visto en clase en el eje de las preguntas de la tabla número 1, sin embargo, en la construcción de dicho esquema se evidenció que los estudiantes no tenían muy claro los componentes que abarcaba la microbiología, lo cual puede deberse a que reflejan concepciones alternativas de tal forma en que se les presentan los saberes científicos como lo menciona Pozo \& Gómez (2006)

"al no presentarse el conocimiento científico como un saber diferente de otras formas de saber, los alumnos tienden a asimilarlos de forma análoga a sus otras fuentes de conocimiento científico y donde, los modelos científicos se mezclan en aquellos ámbitos del discurso cotidiano con referentes comunes" (p. 16).

Por ello, en la segunda sesión se hizo una introducción teórica del tema y luego de la práctica de laboratorio, donde plasmaron lo observado a través de los dibujos.

En las temáticas II, III y IV se categorizaron los resultados como descriptivos ya que los estudiantes hicieron la identificación a nivel de interacción de los microorganismos con su medio, reconociendo la importancia de estos organismos en su medio natural y su uso antrópico. En la práctica de laboratorio también se evidenció el reconocimiento de estructuras y la función que dicha estructura le proporciona al organismo en su medio; dicha práctica se dificultó debido a que los estudiantes no tenían conocimiento acerca del funcionamiento de las partes del microscopio, sin embargo, si poseían la idea de qué era y para qué servía dicho instrumento; reconociendo así en los estudiantes la importancia del microscopio y del buen uso de este, en el aprendizaje de la microbiología y en la producción de conocimiento científico, pues como afirma Valbuena (2007) unas de las formas de proceder en las ciencias corresponde al trabajo de laboratorio, estas actividades prácticas generan en los estudiantes un mayor interés. En dichas actividades se observó una actitud positiva hacia el desarrollo de las mismas generando en los estudiantes dudas e interés sobre los microorganismos. Tal como afima Hodson (1994) quien cita a Gardner y Gauld (1990):

"los estudiantes normalmente disfrutan cuando trabajan en el laboratorio... no todos de igual-manera, e incluso un estudiante que se deleite puede encontrar algunos de sus aspectos insatisfactorios". (p. 300).

También es importante resaltar la edad de los estudiantes, las cuales están entre 11-12 años de edad, en donde las actividades prácticas atraen una gran atención. En donde el entusiasmo por las actividades disminuye en forma significativo con el aumento de la edad como lo registran Hodson (1994) citando a Lynch y Ndyetabura (1984). 
Bio - grafía. Escritos sobre la Biología y su Enseñanza. ISSN 2027-1034

\section{Edición Extraordinaria. p.p. 957 - 965}

Memorias del IX Encuentro Nacional de Experiencias en Enseñanza de la Biología y la Educación Ambiental. IV Congreso Nacional de Investigación en Enseñanza de la Biología.

En el marco de la práctica de laboratorio, se propiciaron las habilidades manipulativas que favorecen el desarrollo personal y autónomo de cada estudiante, teniendo encuentros de participación activa en donde los estudiantes presentaron tanto la relación de los conceptos concebidos previamente y su asociación con los nuevos (MEC, 2006); de este modo los estudiantes identificaron las estructuras observadas en las muestras con aquello que se observa a nivel macroscópico, como las hifas y conidios de los hongos, presentes en la descomposición de los alimentos como el pan, esto evidencia lo descrito por López \& Tamayo (2012), citando a Osorio (2004), donde el trabajo de laboratorio favorece y promueve el aprendizaje de las ciencias, pues le permite al estudiante cuestionar sus saberes y confrontarlos con la realidad.

En el enfoque de los laboratorios coincidimos con lo descrito por Hodson (1994), en la idea del valor de la práctica de la ciencia, en donde esta estrategia le permite una fluidez con la cotidianidad al aprendizaje de las ciencias; en nuestro caso, la interacción de primera mano con los microorganismos les permitió desarrollar una relación con su cotidianidad y la presencia de los mismos en actividades diarias como la descomposición de los alimentos y la presencia de algunos de dichos organismos en el agua.

En este sentido las actividades prácticas permiten un acercamiento a la comunidad científica de modo tal que evidencia la forma de hacer ciencia y su capacidad de analizar los fenómenos. Estas prácticas aportan a la construcción de una visión sobre la ciencia, en donde los estudiantes entienden y se sumergen en la disciplina reconociendo las posibles variables, incentivando la investigación científica de los estudiantes (Hodson, 1994).

Las temáticas II, IV, V y VII se categorizaron como funcional, los estudiantes reconocen la importancia y funcionalidad de las estructuras identificadas en prácticas anteriores así como de su funcionalidad en la biología de los microorganismos. Dichas estructuras son tomadas como datos los cuales al ser funcionales sirven para facilitar otros aprendizaje más significativos (Pozo \& Gómez, 2006).

En este sentido la importancia de las diferentes prácticas permiten en los estudiantes reconocer e identificar las características de los microorganismos tanto a nivel micro como macro de tal manera que puedan relacionar dichas características con su entorno, como lo menciona Rozo (2011), las actividades prácticos se constituyen y se construyen en la dimensión social de los alumnos explicando los fenómenos naturales y del contexto.

La elaboración de los alumnos del concepto de microbiología a partir de estructuras previas como el conocimiento cotidiano puede permitir una mejor concepción de la ciencia, como lo plantea el objetivo del aprendizaje significativo en el cual se exponen los conocimientos previos en interacción con los materiales de aprendizaje y así darle sentido, modificando dichos conocimientos previos con el fin de que surja un nuevo conocimiento; sin embargo, con mayor frecuencia de lo que la explicación ausbeliana haría suponer, cuando los alumnos intentan comprender una nueva situación a partir de sus conocimientos previos, es esa nueva información la que cambia, la que es interpretada en términos de los conocimiento ya adquiridos sin que éstos apenas se modifiquen (Pozo \& Gómez, 2006). Asimismo se resalta la reestructuración de los 
Bio - grafía. Escritos sobre la Biología y su Enseñanza. ISSN 2027-1034

\section{Edición Extraordinaria. p.p. 957 - 965}

Memorias del IX Encuentro Nacional de Experiencias en Enseñanza de la Biología y la

Educación Ambiental. IV Congreso Nacional de Investigación en Enseñanza de la Biología.

conceptos alternos que poseían los alumnos antes del desarrollo teórico-práctico, evidenciándose en las categorías conceptual y funcional, de modo tal que se logró una identificación y caracterización de la biología y estructuras de cada grupo de microorganismos.

Por ello la construcción de dichas prácticas aportan en la constitución y construcción de la ciencia desde el entorno social, así como "la validez de las prácticas experimentales en la constitución de la ciencia, su función independiente de la carga teórica o en equilibrio con ella, y no supeditado a ella solamente en el papel verificacionista o falsacionista" (Cardona, 2013, p. 17), con el que generalmente se ha caracterizado, "...el fundamento de este campo de investigación el cual aplicado desde las primeras etapas escolares los estudiantes deberían experimentar la ciencia de tal manera que les fomente y comprometa a la activa construcción de ideas, de explicaciones y que aumente sus oportunidades para desarrollar la capacidad de 'hacer' ciencia, 'Sobre una realidad científica" (Cardona, 2013, p. 18).

\section{CONCLUSIONES}

Las actividades prácticas son una herramienta facilitadora para el aprendizaje de las ciencias, en el caso específico de la microbiología, donde logró evidenciar que dichas prácticas generan un mayor interés sobre la materia favoreciendo una mayor compresión del tema.

La práctica de laboratorio es un elemento fundamental para la enseñanza y el aprendizaje de la microbiología y la ciencia, en la cual se genera un contacto directo con los microorganismos, permitiendo a los estudiantes identificar, reconocer y contextualizar lo adquirido en las prácticas.

Las prácticas de laboratorio asimismo representan dificultades si no se hace refuerzo en el componente teórico.

\section{REFERENCIAS BIBLIOGRÁFICAS}

Banet, E (2000). La enseñanza y el aprendizaje del conocimiento biológico. En: Perales, F. y Cañal, P. Coomp). Didáctica de las Ciencias experimentales, Editorial Marfil. Alcoy, España. pp. 449-478.

Cardona, F. (2013). Las prácticas de laboratorio como estrategia didáctica. Universidad Del Valle Instituto De Educación Y Pedagogía Licenciatura Básica En Ciencias Naturales Con Énfasis En Medio Ambiente Santiago De Cali.

Del Carmen, L. (2000). Los trabajos prácticos. En: Perales Francisco Javier, Cañal de León, Pedro. Didáctica de las ciencias experimentales. Teoría y práctica de la enseñanza de las ciencias. Alcoi: Marfil. 
Bio - grafía. Escritos sobre la Biología y su Enseñanza. ISSN 2027-1034

\section{Edición Extraordinaria. p.p. 957 - 965}

Memorias del IX Encuentro Nacional de Experiencias en Enseñanza de la Biología y la Educación Ambiental. IV Congreso Nacional de Investigación en Enseñanza de la Biología.

Fonseca, G. (2012) La construcción del conocimiento didáctico del contenido en profesores de biología en formación a través del diseño e implementación de unidades didácticas. III Congreso Internacional y VII Nacional de Investigación en educación, pedagogía y formación docente (Capítulo de Memoria) Pp. 1854-1869.

Hodson, D. (1994). Hacia un enfoque más crítico del trabajo de laboratorio, (p.p. 305). The Ontario Institute for Studies in Education, Toronto (Canadá).

Jiménez A., M. P., Caamaño, A, Oñorbe, A., Pedrinaci, E. \& de Pro, A. (2003). Enseñar Ciencias, Primera edición, Barcelona. España. 240 pp.

López Rua, Ana Milena; Tamayo Alzate, Óscar Eugenio. Las Prácticas De Laboratorio En La Enseñanza De Las Ciencias Naturales Revista Latinoamericana De Estudios Educativos (Colombia), Vol. 8, Núm. 1, Enero-Junio, 2012, Pp. 145-166 Universidad De Caldas Manizales, Colombia.

Novak Joseph D. (1998). Conocimiento y aprendizaje, los mapas conceptuales como herramientas facilitadoras. Madrid. Editorial Alianza.

Pozo J. \& Gómez A., (2006); Aprender y enseñar ciencia. Del conocimiento cotidiano al conocimiento científico. Ediciones Morata, S.L., Madrid, España.

Rozo, J. (2011). Trabajo Práctico: recurso que propicia el aprendizaje significativo sobre diversidad y ecología microbiana en estudiantes de grado cuarto del colegio Champagnat de Bogotá. Revista Bio-grafía: Escritos sobre la Biología y su Enseñanza Universidad Pedagógica Nacional, Bogotá, Colombia, pp 1-18.

Torres, C. (1998). Estrategias y técnicas de investigación cualitativa. Facultad de ciencias sociales y humanas de UNAD. Bogotá, Colombia.

Valbuena, E. (2007). El Conocimiento Didáctico del Contenido Biológico. Estudio de las concepciones disciplinares y didácticas de futuros docentes de la Universidad Pedagógica Nacional (Colombia). Universidad Complutense de Madrid. 